\title{
Ultrasonic and Spectral Studies on Hydrogen Bonded Complexes of Aromatic Aldehydes and $\mathrm{N}$-Methylaniline in $\mathrm{n}$-Hexane
}

\author{
R. Kumar ${ }^{1}$, B.S. Santhi ${ }^{2}$, Vijayakanth ${ }^{3}$ and V. Kannappan ${ }^{2, *}$ \\ ${ }^{1}$ Department of Physics, The New College, Chennai 600 014, India \\ ${ }^{2}$ Department of Chemistry, Presidency College, Chennai 600 005, India \\ ${ }^{3}$ Department of Physics, D.G. Vaishnav College, Chennai 600 106, India
}

\begin{abstract}
Ultrasonic and UV-spectral studies have been carried out for three ternary systems containing $\mathrm{N}$-methylaniline (NMANI) and three structurally different aromatic aldehydes, benzaldehyde (BA), cinnamaldehyde (CA) and salicylaldehyde(SA) in $\mathrm{n}$-hexane medium at $303.15 \mathrm{~K}$ and at atmospheric pressure. Acoustical parameters are computed from the measured values of ultrasonic velocity, density and dynamic viscosity. The variation of acoustical parameters in the concentration range investigated establishes complex formation through intermolecular hydrogen bonding between aldehyde and $\mathrm{N}$-methylaniline. The existence of strong aldehyde-amine interaction is also confirmed through the recorded UV-Visible absorption spectra with Benesi-Hildebrand theory at $303.15 \mathrm{~K}$. The formation constants of the hydrogen bonded complexes are determined by spectroscopic and ultrasonic methods and compared. These values computed by two different methods are comparable and follow similar trend. The trend in the formation constants is discussed based on structures of the component molecules and correlate with computed molecular properties.
\end{abstract}

Keywords: Aldehyde-amine H-bonded complex, stability constants, structural effect.

\section{INTRODUCTION}

In recent years, the ultrasonic technique is widely employed in the detection of charge transfer complexes $[1,2]$ and in the determination of stability constants and thermodynamic properties of the complexes [3-5]. Hydrogen bond plays important role in strengthening of molecular interactions and stabilizing supra-molecular complexes. Study of hydrogen bonded systems is important and useful as hydrogen bond plays important role in chemical, physical and biological processes. Organic compounds containing electronegative groups can interact with compounds containing active hydrogen through hydrogen bond [6]. This type of hydrogen bond plays important role in the stability of biologically important molecules [7]. Carbonyl group is part of several biologically important molecules such as proteins, lipids and hormones. Amines in pure state are self-associated through inter molecular hydrogen bonds. They are both $\pi$-as well as n-electron donors which allow them to have specific interactions with other electron deficient molecules. The complexes formed between esters and amines, phenols and ketones have been attributed to the hydrogen bonding between electron-rich carbonyl oxygen and active hydrogen. Higuchi and his coworkers [8] have investigated the complex formation of caffeine with a number of acidic drugs. They attributed the interaction

*Address correspondence to this author at the Department of Chemistry, Presidency College, Chennai 600 005, India; Tel: +91 9840821026;

E-mail: vkannappan48@gmail.com between caffeine and drug todipole-dipole force or hydrogen bonding between the polarized carbonyl groups of caffeine and the hydrogen atom of the acid. Similarly, in the formation of 1:1 complex between $p$ benzoquinone and hydroquinone, hydrogen bond exists between the donor and acceptor components [9]. The present investigation is aimed at detection of the possibility of specific interaction between secondary aromatic amine, $\mathrm{N}$-methylaniline and aromatic aldehydes in $\mathrm{n}$-hexane medium through ultrasonic and UV-Visible spectroscopic techniques.

\section{EXPERIMENTAL}

$\mathrm{N}$-Methylaniline, benzaldehyde and the solvent $\mathrm{n}$ hexane used were AnalaR grade MERCK samples with the estimated purity of $>99.8 \%$. Cinnamaldehyde and salicylaldehyde were of spectrochem purity supplied by SD fine Chemicals, India. The reagents were purified before use in accordance with the procedure described elsewhere $[10,11]$ and the boiling points of the four compounds agreed well with the literature values. A single crystal variable path ultrasonic interferometer (Model F-81) operating at $2 \mathrm{MHz}$ frequency supplied by Mittal Enterprises, India was used in the measurement of ultrasonic velocity of liquid mixtures. The precision of the speed of sound measurements was estimated to be $\pm 0.1 \mathrm{~m} / \mathrm{s}$. The estimated uncertainty is $0.2 \mathrm{~m} / \mathrm{s}$. The instrument was calibrated by measuring the velocity of triple distilled water, carbon tetrachloride at $298 \mathrm{~K}$ and dimethyl sulfoxide at $303 \mathrm{~K}$ and these values are comparable with literature values [12-14]. A $10 \mathrm{ml}$ 
specific gravity bottle and a single pan digital balance of Shimadzu make with an accuracy of $\pm 0.1 \mathrm{mg}$ were used to determine the density of the solutions. Ostwald's viscometer was used to determine the viscosity, in which the flow time of the solution was measured through a digital stop clock of accuracy \pm $0.01 \mathrm{~s}$. In all cases, the experiments were performed generally at least in six replicates for each composition and the results were averaged. The reproducibility in mole fraction was within \pm 0.0002 units. The temperature of the samples was maintained constant to an accuracy of $\pm 0.1 \mathrm{~K}$ by digitally controlled thermostatic water bath. UV-visible spectra were recorded in a Shimadzu UV-1650 model spectrophotometer with quartz cell of $1 \mathrm{~cm}$ optical path length. The solvent, n-hexane was used for the base line correction. The absorption spectra were recorded in the wavelength region of $200-700 \mathrm{~nm}$ with the scanning rate of $0.2 \mathrm{~nm} / \mathrm{s}$ and a slit width of $1 \mathrm{~cm}$.

From the measured values of ultrasonic velocity $(u)$, density ( $r$ ) and viscosity (h), various acoustical parameters were calculated using the standard equations reported in the literature [12]. The stability constants of the complexes using ultrasonic velocity values by employing Kannappan equation $[4,5]$ and

Table 1: Ultrasonic velocity $u$, density $\rho$, viscosity $\eta$, adiabatic compressibility $\kappa$, acoustic impedance $Z$, free length Lf, internal pressure $\pi$ free volume $V_{f}$, cohesive energy CE and interaction parameter $\chi$ vales for solutions containing equimolar concentration of $\mathrm{BA}$ and $\mathrm{N}$-methylaniline at $303.15 \mathrm{~K}$

\begin{tabular}{|c|c|c|c|c|c|c|c|c|c|}
\hline $\begin{array}{c}\text { Conc., }{ }^{\star} / 10^{-4} \\
\text { M }\end{array}$ & $\begin{array}{c}\mathbf{u} \\
\mathbf{m ~ s} \mathbf{s}^{-1}\end{array}$ & $\begin{array}{c}\rho \\
\mathrm{kg} \mathrm{m}^{-3}\end{array}$ & $\begin{array}{l}\eta / 10^{-4} \\
\mathrm{Nsm}^{-2}\end{array}$ & $\begin{array}{c}\mathrm{\kappa} / 10^{-9} \\
\mathrm{~kg}^{-1} \mathrm{~ms}^{-2}\end{array}$ & $\begin{array}{c}\mathrm{Z} / 10^{5} \\
\mathrm{~kg} \mathrm{~m}^{-2} \mathrm{~s}^{-1}\end{array}$ & $\begin{array}{l}\text { Lf } \\
A^{\circ}\end{array}$ & $\begin{array}{c}\pi / \\
\text { atm }\end{array}$ & $\begin{array}{c}\text { CE } \\
\mathrm{kJ} \mathrm{mole}^{-1}\end{array}$ & $\chi^{/ 10^{-2}}$ \\
\hline 1 & 1043.2 & 648.6 & 3.36 & 1.42 & 6.77 & 0.747 & 2344 & 31.5 & -2.58 \\
\hline 2 & 1043.6 & 649.0 & 3.38 & 1.41 & 6.77 & 0.746 & 2350 & 31.6 & -2.51 \\
\hline 3 & 1044.0 & 649.8 & 3.39 & 1.41 & 6.78 & 0.746 & 2355 & 31.6 & -2.43 \\
\hline 4 & 1044.4 & 650.0 & 3.39 & 1.41 & 6.79 & 0.745 & 2356 & 31.6 & -2.36 \\
\hline 5 & 1045.6 & 650.4 & 3.40 & 1.41 & 6.80 & 0.744 & 2359 & 31.7 & -2.14 \\
\hline 6 & 1046.0 & 650.8 & 3.41 & 1.41 & 6.81 & 0.744 & 2362 & 31.7 & -2.06 \\
\hline 7 & 1046.8 & 651.1 & 3.41 & 1.40 & 6.81 & 0.743 & 2363 & 31.7 & -1.92 \\
\hline 8 & 1047.2 & 651.2 & 3.42 & 1.40 & 6.82 & 0.742 & 2365 & 31.7 & -1.84 \\
\hline 9 & 1048.0 & 652.6 & 3.43 & 1.40 & 6.84 & 0.741 & 2372 & 31.7 & -1.70 \\
\hline 10 & 1049.6 & 653.4 & 3.45 & 1.39 & 6.86 & 0.740 & 2378 & 31.8 & -1.40 \\
\hline
\end{tabular}

Solvent: $\mathrm{n}$-hexane $\mathrm{T}=303.15 \mathrm{~K}$.

Table 2: Ultrasonic velocity $u$, density $\rho$, viscosity $\eta$, adiabatic compressibility $\kappa$, acoustic impedance $Z$, free length Lf, internal pressure $\pi$ free volume $V_{f}$, cohesive energy CE and interaction parameter $\chi$ values for solutions containing equimolar concentration of $\mathrm{CA}$ and $\mathrm{N}$-methylaniline at $303.15 \mathrm{~K}$

\begin{tabular}{|c|c|c|c|c|c|c|c|c|c|}
\hline $\begin{array}{c}\text { Conc., }{ }^{*} / 10^{-4} \\
\mathbf{M}\end{array}$ & $\begin{array}{c}u \\
\mathrm{~m} \mathrm{~s}^{-1}\end{array}$ & $\begin{array}{c}\rho \\
\mathrm{kg} \mathrm{m}^{-3}\end{array}$ & $\begin{array}{l}\eta / 10^{-4} \\
\mathrm{Nsm}^{-2}\end{array}$ & $\begin{array}{c}\mathrm{\kappa} / 10^{-9} \\
\mathrm{~kg}^{-1} \mathrm{~ms}^{-2}\end{array}$ & $\begin{array}{c}\mathrm{Z} / 10^{5} \\
\mathrm{~kg} \mathrm{~m}^{-2} \mathrm{~s}^{-1}\end{array}$ & $\begin{array}{l}\text { Lf } \\
A^{\circ}\end{array}$ & $\begin{array}{c}\pi / \\
\operatorname{atm}\end{array}$ & $\begin{array}{c}\text { CE } \\
\mathrm{kJ} \mathrm{mole}^{-1}\end{array}$ & $\chi / 10^{-2}$ \\
\hline 1 & 1037.6 & 646.4 & 3.40 & 1.44 & 6.71 & 0.752 & 2358 & 31.8 & -3.62 \\
\hline 2 & 1038.4 & 647.8 & 3.41 & 1.43 & 6.73 & 0.751 & 2365 & 31.9 & -3.47 \\
\hline 3 & 1039.2 & 647.9 & 3.41 & 1.43 & 6.73 & 0.750 & 2365 & 31.9 & -3.33 \\
\hline 4 & 1040.8 & 648.0 & 3.42 & 1.42 & 6.74 & 0.749 & 2365 & 31.9 & -3.03 \\
\hline 5 & 1041.2 & 649.4 & 3.43 & 1.42 & 6.76 & 0.748 & 2371 & 31.9 & -2.96 \\
\hline 6 & 1042.0 & 649.6 & 3.43 & 1.42 & 6.77 & 0.747 & 2371 & 31.9 & -2.81 \\
\hline 7 & 1043.2 & 649.8 & 3.43 & 1.41 & 6.78 & 0.746 & 2371 & 31.9 & -2.59 \\
\hline 8 & 1044.0 & 650.0 & 3.44 & 1.41 & 6.79 & 0.745 & 2373 & 31.9 & -2.44 \\
\hline 9 & 1045.2 & 651.8 & 3.45 & 1.41 & 6.80 & 0.744 & 2376 & 31.9 & -2.22 \\
\hline 10 & 1046.0 & 651.7 & 3.45 & 1.40 & 6.82 & 0.743 & 2380 & 31.9 & -2.08 \\
\hline
\end{tabular}

Solvent: $\mathrm{n}$-hexaneT=303.15 K. 
using the absorbance values of UV-visible spectra by employing Benesi-Hildebrand equationwere computed as reported in our earlier work [15].

Molecular orbital calculations are carried out for aldehydes to determine the complexation site and to correlate formation constants with the molecular properties. MNDO method gives satisfactory electronic charge distribution. The gross atomic charge on the carbonyl oxygen atom of different aldehyde molecules is calculated by MNDO/3 method. The optimization of the molecular geometry has been done using GAMMESS software. The other molecular properties dipole moment and ionization potential values are also computed by MNDO/3 method.

\section{RESULTS AND DISCUSSION}

\subsection{Acoustical Parameters}

The measured properties, ultrasonic velocity $(u)$, density $(\rho)$, and viscosity $(\eta)$ and the calculated acoustical parameters for the three ternary systems at 303.15K are summarized in Tables 1-3. The graphical representation for the variations of ultrasonic velocity $(\mathrm{u})$ and free volume $\left(\mathrm{V}_{\mathrm{f}}\right)$ for solutions containing equimolar concentration of solutes are depicted in Figures 1 and 2. An inspection of the acoustical parameters in Tables 1-3 reveals that the ultrasonic velocity increases with increase in concentration in the three ternary systems. The variation in the ultrasonic velocity with concentration (Figure 1) is similar in all the three systems. The increase in velocity may be attributed to strong solute-solute interactions. It was reported that the strength of molecular interactions in liquid mixtures becomes high where the velocity maximum occurs [16]. The highly polar nature of the carbonyl group of aldehyde may disrupt the dipolar association in pure amine leading to the possibility of unlike molecular attraction. The steep increase in the ultrasonic velocity may be an indication of stronger molecular interactions such as dipole-dipole or complex formation through intermolecular hydrogen bond [17]. It may be pointed out that the variation in ultrasonic velocity is small as the solutions are very dilute.

The trend in adiabatic compressibility and free length with concentration shows reverse trend as that of ' $u$ ' and ' $\rho$ '. A continuous decrease in adiabatic compressibility $(\kappa)$ and intermolecular free length $(\mathrm{Lf})$ supports the existence of strong interactions between $\mathrm{N}$-methylaniline and aldehyde in the liquid mixtures which lead to the increasing compactness of the systems. The rapid decrease of ' $\kappa$ ' indicates the formation of a large number of tightly bound systems. Such a reduction in the ' $\kappa$ ' brings the molecules to a close packing, resulting in a decrease ofthe free length 'Lf' [18].

The internal pressure is a measure of the resultant force of attraction between the interacting components in liquid mixtures and it can be used to assess intermolecular interaction. The internal pressure

Table 3: Ultrasonic velocity $u$, density $\rho$, viscosity $\eta$, adiabatic compressibility $\kappa$, acoustic impedance $Z$, free length Lf, internal pressure $\pi$ free volume $V_{f}$, cohesive energy CE and interaction parameter $\chi$ values for solutions containing equimolar concentration of SA andN-Methylaniline at $303.15 \mathrm{~K}$

\begin{tabular}{|c|c|c|c|c|c|c|c|c|c|}
\hline $\begin{array}{c}\text { Conc., }{ }^{*} / 10^{-4} \\
\text { M }\end{array}$ & $\begin{array}{c}\mathbf{u} \\
\mathrm{m} \mathrm{s}^{-1}\end{array}$ & $\begin{array}{c}\rho \\
\mathrm{kg} \mathrm{m}^{-3}\end{array}$ & $\begin{array}{l}\eta / 10^{-4} \\
\mathrm{Nsm}^{-2}\end{array}$ & $\begin{array}{c}\mathrm{K} / 10^{-9} \\
\mathrm{~kg}^{-1} \mathrm{~ms}^{-2}\end{array}$ & $\begin{array}{c}\mathrm{Z} / 10^{5} \\
\mathrm{~kg} \mathrm{~m}^{-2} \mathrm{~s}^{-1}\end{array}$ & $\begin{array}{l}\text { Lf } \\
A^{\circ}\end{array}$ & $\begin{array}{c}\pi / \\
\text { atm }\end{array}$ & $\begin{array}{c}\text { CE } \\
\mathrm{kJ} \mathrm{mole}^{-1}\end{array}$ & $\chi^{/ 10^{-2}}$ \\
\hline 1 & 1046.0 & 657.0 & 3.59 & 1.39 & 6.87 & 0.740 & 2438 & 32.4 & -2.05 \\
\hline 2 & 1046.4 & 657.1 & 3.59 & 1.39 & 6.87 & 0.740 & 2441 & 32.4 & -1.98 \\
\hline 3 & 1046.8 & 657.6 & 3.60 & 1.39 & 6.88 & 0.739 & 2444 & 32.4 & -1.91 \\
\hline 4 & 1047.2 & 657.9 & 3.61 & 1.39 & 6.89 & 0.739 & 2447 & 32.5 & -1.84 \\
\hline 5 & 1048.0 & 658.0 & 3.61 & 1.38 & 6.89 & 0.738 & 2449 & 32.5 & -1.69 \\
\hline 6 & 1048.4 & 658.1 & 3.61 & 1.38 & 6.89 & 0.738 & 2447 & 32.5 & -1.62 \\
\hline 7 & 1048.8 & 658.2 & 3.62 & 1.38 & 6.90 & 0.737 & 2449 & 32.5 & -1.54 \\
\hline 8 & 1049.6 & 659.0 & 3.62 & 1.38 & 6.91 & 0.736 & 2452 & 32.5 & -1.39 \\
\hline 9 & 1050.4 & 661.0 & 3.64 & 1.37 & 6.94 & 0.735 & 2462 & 32.5 & -1.25 \\
\hline 10 & 1051.6 & 661.9 & 3.65 & 1.37 & 6.94 & 0.733 & 2468 & 32.5 & -1.02 \\
\hline
\end{tabular}

Solvent: $n$-hexane $T=303.15 \mathrm{~K}$. 


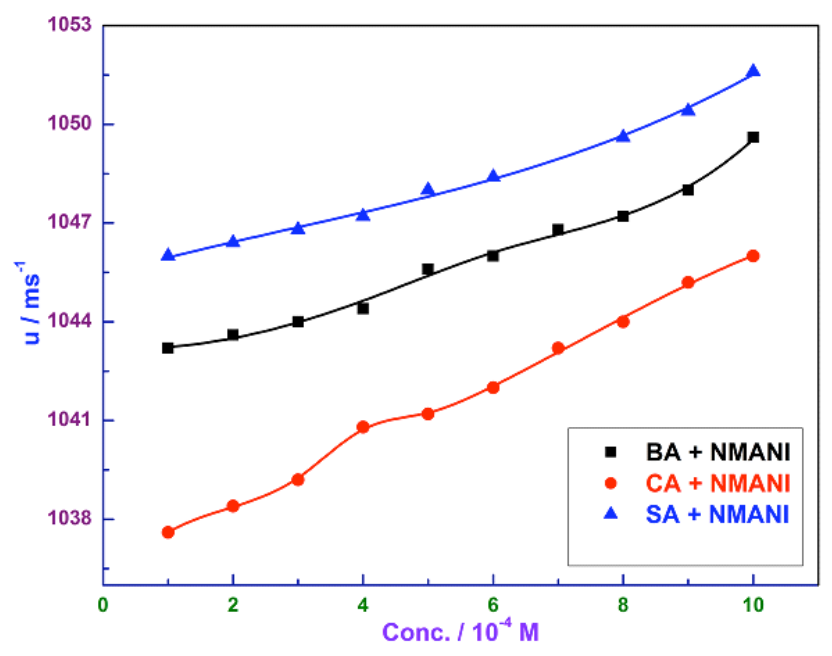

Figure 1: Plot of ultrasonic velocity versus equimolar concentration of $\mathrm{N}$-methylaniline and three aldehydes in $\mathrm{n}$ hexane solution at $303.15 \mathrm{~K}$.

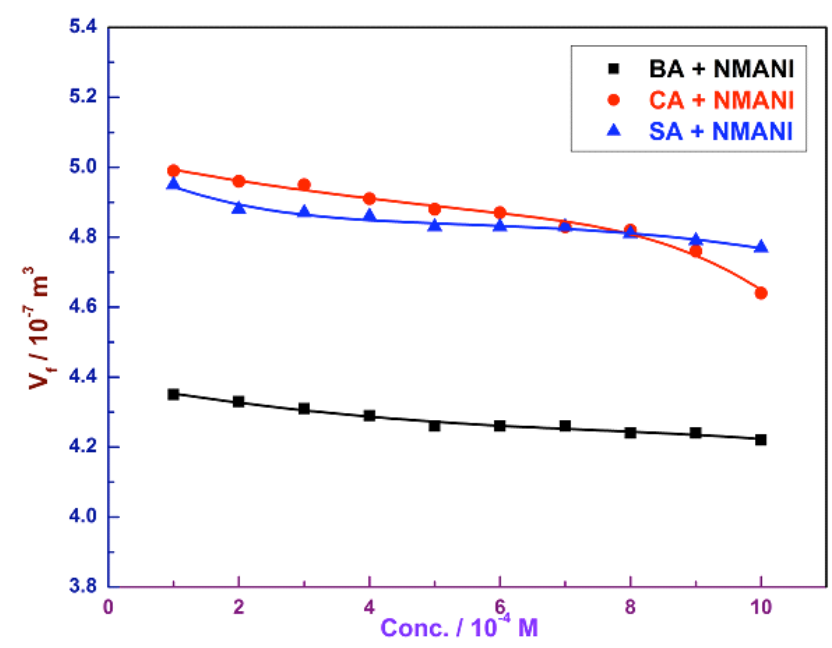

Figure 2: Plot of free volume versus equimolar concentration of $\mathrm{N}$-methylaniline and three aldehydes in $\mathrm{n}$-hexane solution at $303.15 \mathrm{~K}$.

increases as the concentration increases in all the three systems investigated. A reverse trend is observed in the variations of free volume $\left(\mathrm{V}_{\mathrm{f}}\right)$ which is indicated in Figure 2. The increase in internal pressure with concentration at the temperature of investigation clearly explains the growing molecular association through stronger dipole-dipole type of interaction against thermal agitation of molecules. The decreasing trend of $V_{f}$ with concentration suggests the tightly bound system of molecules inside the shield, which may be brought about by the growing magnitude of solute-solute interaction [19]. The trend in the values of free volume with concentration suggests that there may be strong intermolecular hydrogen bonds between the amine and aldehyde.
The magnitude of cohesive energy (CE) is almost constant for all the systems and variation in CE with concentration is not significant. The magnitude of CE indicates the presence of strong dipole-dipole attraction, probably intermolecular hydrogen bonding in the ternary systems. Molecular interaction parameter in a system is a measure of deviation from ideal behavior and hence it gives the strength of molecular interaction in binary and ternary systems. The negative values of interaction parameter also reveal the presence of strong interactions between carbonyl compounds and NMANI. The acoustic impedance is almost constant in the concentration range studied and also almost similar values are obtained in the three systems suggesting similar type of intermolecular interaction in these systems. Thus perusal of the combined variations of the measured properties and the computed acoustical parameters clearly reveal the possibility of strong interactions between the aldehyde and amine molecules. In order to confirm the existing interaction and the structural effect of these molecules taking part in the interaction, UV-Visible spectroscopic method has been employed.

\subsection{Analysis of UV-Visible Spectra}

The recorded UV-Visible spectra for the n-hexane solutions of cinnamaldehyde and salicylaldehyde

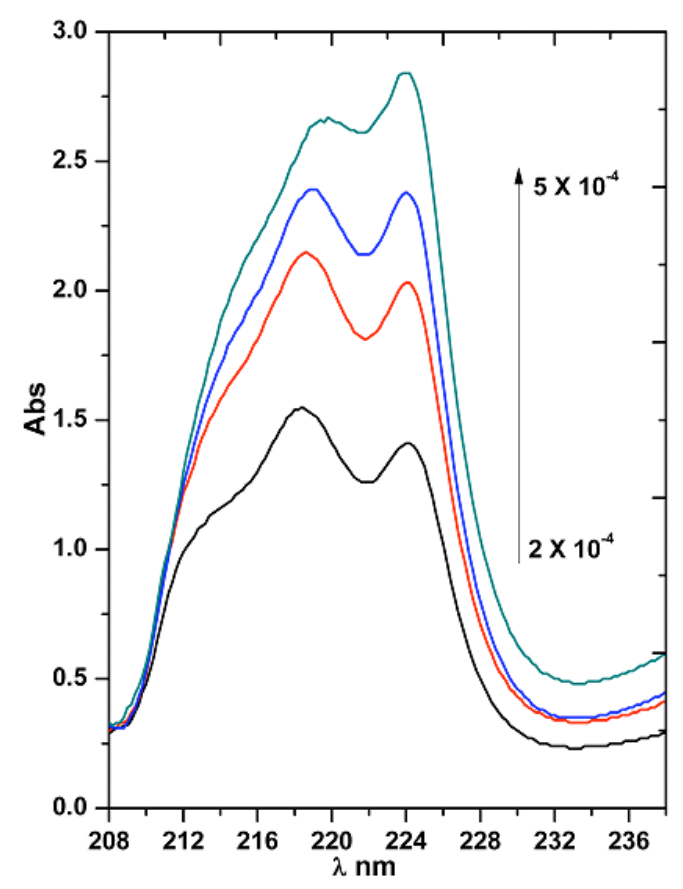

Figure 3: UV-Vis spectra for the variation of wavelength I versus absorbance for $\mathrm{N}$-methylaniline and $\mathrm{CA}$ in $\mathrm{n}$-hexane medium at $303.15 \mathrm{~K}$. The concentration of amine was kept constant at $1 \times 10^{-5} \mathrm{M}$ and the concentration of aldehyde was varied in excess as shown. 


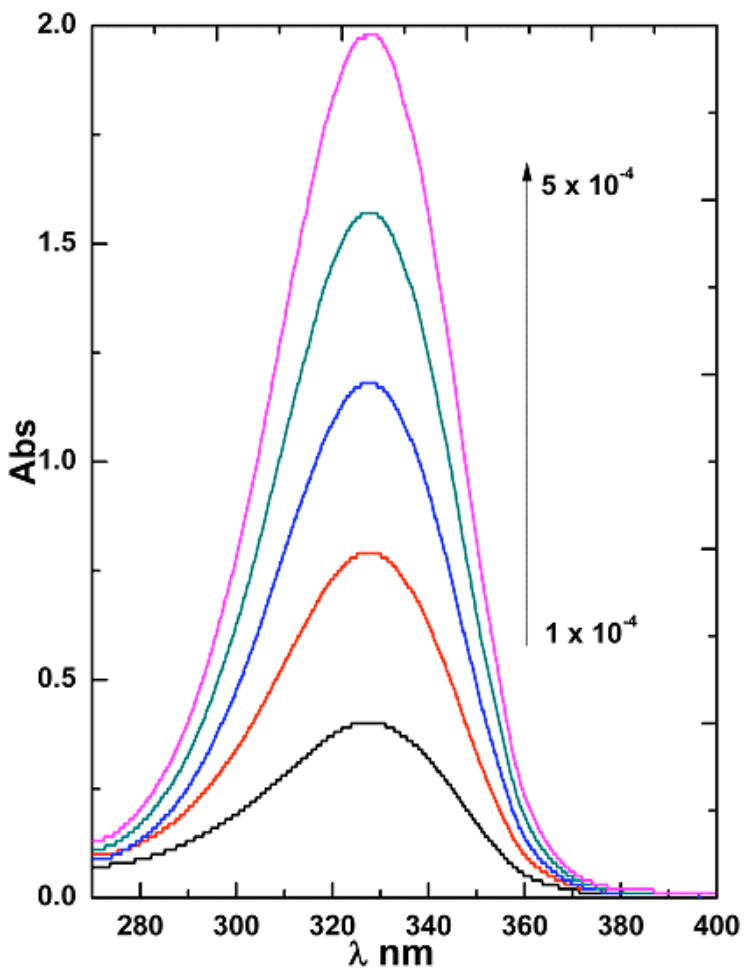

Figure 4: Recorded UV-Vis spectra for the variation of wavelength I versus absorbance for $\mathrm{N}$-methylaniline and SA in $\mathrm{n}$-hexane medium at $303.15 \mathrm{~K}$. The concentration of amine was kept constant at $1 \times 10^{-5} \mathrm{M}$ and the concentration ofaldehyde, was varied in excess as shown.

system with NMANI are shown in Figures 3 and 4 . It can be seen that the aldehyde absorbs at $243 \mathrm{~nm}$ due to $\pi-\pi^{*}$ transition. The $n-\pi^{*}$ absorption occurs at a shorter wavelength which is not shown in the spectra. $\mathrm{N}$-Methylaniline (NMANI) gives a broad and weak absorption at $294.0 \mathrm{~nm}$ and a sharp and strong absorption at $242.4 \mathrm{~nm}$. Further, the wavelengths of absorption for the three CT complexes lie at 239.8, 223.6 and $328.6 \mathrm{~nm}$ respectively for the three aldehydes benzaldehyde, cinnamaldehyde and salicyaldehyde with NMANI in n-hexane. In this ternary mixture the aldehyde is hydrogen bonded to NMANI which is a polar molecule containing active hydrogen. Generally, the $\pi-\pi^{*}$ transition is shifted to shorter wave length in more polar environment due to stability of the ground state in polar environment [20, 21]. Therefore, the complex absorbs at a shorter wave length than those of pure components. Similar observations are obtained in the other two systems. In order to determine the formation constants of these complexes Benesi-Hildebrand method was employed [22]. In this method, [NMANI] was kept constant and the [aldehyde] was varied and taken in excess. In the present investigation, the concentration of amine was kept constant at $1.5 \times 10^{-5} \mathrm{M}$ and the concentration of aldehyde was taken 10 to 50 times and varied. The absorbance values are calculated at this wave length of maximum absorption and used to make BenesiHildebrand plots (Figure 5). The spectra were recorded after making baseline correction with the solvent n-hexane.

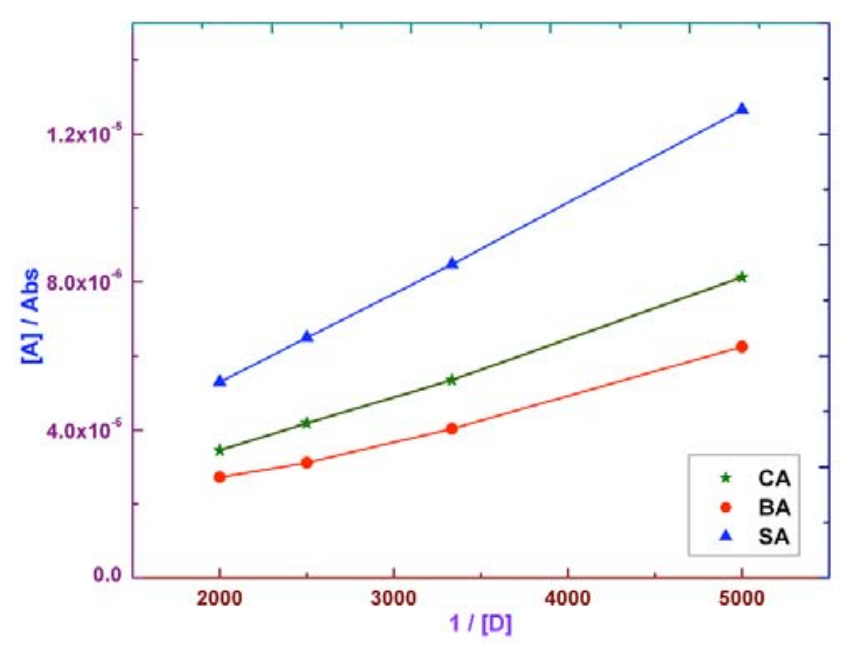

Figure 5: Benesi-Hildebrand plot drawn between reciprocal of concentration of aldehyde [D] versus ratio of oncentration of NMANI, [A] to absorbance for aldehyde and NMANI in $\mathrm{n}$-hexane medium at $303.15 \mathrm{~K}$.

\subsection{Structural Effect on Stability Constant}

The thermodynamic stability of the hydrogen bonded charge transfer complexes formed between the aromatic aldehydes and aromatic secondary amine can be compared by determining the stability constants of these complexes at the temperature of investigation. These values were calculated by employing BenesiHildebrand $(\mathrm{B}-\mathrm{H})$ equation with the observed values of optical absorbance in UV spectra. These results along with free energy of formation $\left(\Delta G^{*}\right)$, maximum absorption wavelength $\left(\lambda_{\max }\right)$ and molar absorptivities $\left(\varepsilon_{\max }\right)$ of the complexes are listed in Table 4 . The Benesi-Hildebrand plot between reciprocal of donor concentration (1/ [D]) versus acceptor concentration / absorbance $([\mathrm{A}] / \mathrm{Abs})$ for the determination of stability constant $(\mathrm{K})$ and molar extinction coefficient $(\varepsilon)$ of the charge transfer complexes is shown in Figure 5 . The B$\mathrm{H}$ plots obtained for the charge transfer complexes were found to be linear with a positive slope and intercept. A linear B-H plot is an indication of the formation of 1:1 complexes between aldehydes and amine [23].

The stability constant values computed by ultrasonic and free energy of formation are listed in Table 4. It is found that the stability constant depends on the 
Table 4: Stability constant $\mathrm{K}$, free energy of formation $\Delta \mathrm{G}^{*}$, relaxation time $\tau$, wave length of maximum absorption $\lambda_{\max }$ and molar extinction coefficient $\mathrm{e}$ values for certain hydrogen bonded complexes of aldehydes with $\mathrm{N}$ methylaniline in nhexane medium obtained by ultrasonic and UV Spectrometric techniques at $303.15 \mathrm{~K}$

\begin{tabular}{|c|c|c|c|c|c|c|c|}
\hline \multirow{2}{*}{ Compounds } & \multicolumn{3}{|c|}{ Ultrasonic method } & \multicolumn{4}{c|}{ UV-Visible Spectroscopic method } \\
\cline { 2 - 9 } & $\mathbf{K} / \mathbf{m o l}^{-1}$ & $\left.\Delta \mathbf{G}^{*} / \mathbf{k J m o l}^{-1}\right)$ & $\tau / \mathbf{1 0 - 1 3} \mathbf{~ s}$ & $\mathbf{K} / \mathbf{m o l}-\mathbf{1}$ & $\Delta \mathbf{G}^{*} /\left(\mathbf{k J m o l}^{-1}\right)$ & $\lambda_{\max } /(\mathbf{n m})$ & $\boldsymbol{\varepsilon} / \mathbf{1 0}^{\mathbf{6}}\left(\mathbf{m}^{\mathbf{2}} \mathbf{m o l}^{-1}\right)$ \\
\hline \hline BA & 189.2 & -13.21 & 6.4 & 190.73 & -12.73 & 239.8 & 3.35 \\
\hline CA & 195.9 & -13.30 & 6.5 & 202.66 & -12.94 & 223.6 & 1.84 \\
\hline SA & 202.3 & -13.38 & 6.7 & 225.82 & -13.71 & 328.6 & 1.85 \\
\hline
\end{tabular}

structure of the aldehyde. A perusal of the values presented in Table 4 reveals that the stability constant for the benzaldehyde system is the least whereas for salicyaldehyde system it is the highest among the three systems investigated. This observation can be explained as follows:

Carbonyl compounds are dipolar compounds and they are weakly associated liquids in pure state but they are associated with molecules of other components like amines and hydroxy derivatives in mixtures through strong intermolecular hydrogen bond. This can be detected in non-polar medium like $n$ hexane The hydrogen bond is formed between carbonyl oxygen of aldehyde and the hydrogen atom of the amino or $\mathrm{O}-\mathrm{H}$ group. In the present case, the complexation through hydrogen bond occurs between the carbonyl oxygen of aldehyde and the hydrogen atom of the secondary amino group. The type of intermolecular interaction between aldehyde and $\mathrm{N}$ methylaniline is illustrated in Figure 6 . Since the complexation occurs between carbonyl oxygen of aldehyde and hydrogen of amino group in amine, its stability is influenced by the structure of component molecules. Mainly it depends on the availability of electrons on carbonyl oxygen and nitrogen of amino group. To study the influence of structure of aldehydemolecules on the stability of the complex formed between aldehydes and amines, three structurally different aromatic aldehydes were chosen. The stability constants are in the order salicyladehyde $>$ cinnamaldehyde > benzaldehyde. There is extension of conjugation in cinnamaldehyde molecule which favours electron release from aromatic ring to carbonyl oxygen and hence the carbonyl oxygen is richer in electron density than in benzaldehyde. This makes CANMANI complex more stable than BA-NMANI complex. The stability constant values obtained by both acoustic and optical methods are in support of this expectation. In salicyladehyde, there is electron releasing group $\mathrm{O}$ $\mathrm{H}$ is present at ortho position to aldehyde group. This group releases electron by mesomeric effect and makes carbonyl oxygen more electronegative than that in benzaldehyde. Another possible explanation is that amino group in NMANI molecule contains one active hydrogen atom. This may be hydrogen bonded to carbonyl oxygen. The adjacent nitrogen atom may be involved in the secong hydrogen bond with the phenolic hydrogen which is in the vicinity of the carbonyl group. Thus, there are two hydrogen bonds between NMANI and salicylaldehyds and hence NMANI forms more stable complex with salicyladehyde than both benzaldehyde and cinnamaldehyde.

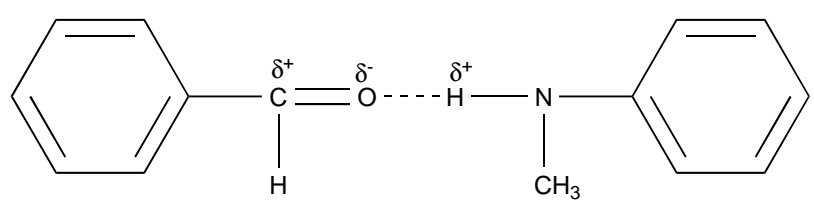

Figure 6: Possible mechanism for the formation of hydrogen bonding between carbonyl oxygen of aldehyde with the imino hydrogen of $\mathrm{N}$-methylaniline.

In order to understand the relation between stability of complex and molecular properties, dipole moment $(\mu)$, ionization energy and net charge on carbonyl oxygen $(\Delta \mathrm{Qt})_{\mathrm{O}}$ are computed by MOPAC calculations. The results are presented in Table $\mathbf{5}$. Hydrogen bond is a strong dipole-dipole interaction and hence the molecule with larger dipole moment can form more stable complex with NMANI. In the present investigation salicylaldehyde has larger dipole moment than the other two aldehydes and hence it forms the most stable complex among the three aldehydes. In the formation of hydrogen bonded complex the molecules involved are polarized and it depends on the ionization energy of the component molecules. Smaller the ionization energy, greater is the ease of polarization and hence it forms more stable complex. In the present investigation salicylaldehyde has the least ionization energy and hence it forms a more stable complex than the other two aldehydes. Considering the net charge on carbonyl oxygen, we expect that cinnamaldehyde forms more stable complex than benzaldehyde. The observed values of formation constants are in support 
Table 5: Log $\mathrm{K}$ values of aldehyde - $\mathrm{N}$-methylaniline complexes along with net electronic charge of carbonyl oxygen $\left(-\Delta \mathbf{Q}_{\mathrm{t}}\right)_{\mathrm{o}}$, polarisability $(\alpha)$, dipole moment $(\mu)$ and ionisation energy $(\mathrm{eV})$ values of aldehydes

\begin{tabular}{|c|c|c|c|c|}
\hline Compounds & $\log K$ & $\left(-\Delta \mathbf{Q}_{\mathrm{t}}\right)_{\mathrm{o}} / 10^{-3}$ & $\mu / D$ & Ionisation Energy, eV \\
\hline BA & 2.277 & -528 & 3.30 & 9.22 \\
\hline $\mathrm{CA}$ & 2.292 & -542 & 3.80 & 8.14 \\
\hline SA & 2.306 & -539 & 4.76 & 8.47 \\
\hline
\end{tabular}

of the expected trend. However, the electron density on carbonyl oxygen of salicylaldehyde is less than that in cinnamaldehyde. On the basis of net charge on carbonyl oxygen of the aldehyde, it is expected that $\mathrm{K}$ value of cinnamaldehyde should be greater than that of salicyladehyde. But reverse trend is observed. This shows that it is not only the net charge on carbonyloxygen which determines the formation constant of the hydrogen bonded complex, but the number of hydrogen bonds that can be formed between the component molecules also determines the stability of these complexes. The negative values of free energy of formation suggest that the complexes are thermodynamically stable and almost close values indicate the formation of similar types of complexes in the three systems. The high values of molar extinction coefficient identifies the presence of Strong hydrogen bonded complexes existing in these systems.

\section{CONCLUSION}

The formation of intermolecular hydrogen bonded complexes between $\mathrm{N}$-methylaniline and three structurally different aromatic aldehydes is established through ultrasonic and spectral studies. Acoustical parameters are calculated for these systems which establish the formation of strong hydrogen bonded complexes between aldehydes and amine. Stability constants are computed for the three complexes by ultrasonic and UV-visible spectroscopic methods. The stability is in the order salicylaldehyde - Nmethylaniline > cinnamaldehyde $\mathrm{N}$-methylaniline > benzaldehyde $\mathrm{N}$-methylaniline. This trend is explained with the electronic effects and the number of hydrogen bonds. The complexes are thermodynamically stable as evidenced from their negative free energy of formation values as well as large molar extinction coefficients. They have almost similar relaxation time which shows that the aldehydes form structurally similar complexes with $\mathrm{N}$-methylaniline.

\section{ACKNOWLEDGEMENT}

One of the authors, B.S. Santhi thanks the University Grants Commission, New Delhi, India for the award of Teacher Fellowship to complete the research work.

\section{REFERENCES}

[1] Oswal SL, Gardas RL, Phalak RP. Densities, speeds of sound, isentropic compressibilities, refractive indices and viscosities of binary mixtures of tetrahydrofuran with hydrocarbons at $303.15 \mathrm{~K}$. J Mol Liq 2005; 116: 109-18. http://dx.doi.org/10.1016/j.molliq.2004.07.081

[2] Ali A, Nain AK, Sharma VK, Ahmad S. Study of molecular interaction in ternary mixtures through ultrasonic speed measurements. Phys Chem Liq 2004; 42: 375-83. http://dx.doi.org/10.1080/00319100410001679882

[3] Kumar R, Swarnalathan S, Mahesh R, Shanmugapriyan B, Kannappan V. Ultrasonic and spectroscopic studies on hydrogen bonded complexes of aromatic amine and aryl ketones inn-hexane at 303.15 K. J Mol Liq 2011; 163: 57-63. http://dx.doi.org/10.1016/j.molliq.2011.07.010

[4] Kumar R, Ulagendran V, Kannappan V, Jayakumar S. Thermo acoustic and spectral investigations of charge transfer interaction between aromatic amine and ketones. Fluid Phase Equlibria 2011; 307: 113-25.

http://dx.doi.org/10.1016/j.fluid.2011.04.019

[5] Kumar R, Jayakumar S, Kannappan V. Thermodynamic properties of donor-acceptor complexes of tertiary amine with aryl ketones in hexane medium. Thermochimica Acta 2012; 536: 15-23.

http://dx.doi.org/10.1016/.t.tca.2012.02.018

[6] Parsons Andrew F. Keynotes in organic chemistry, Blackwell Science Limited, London, Oxford 2003; p. 2.

[7] Cai J, Wang RE, Eds. Protein Interactions, Published by InTech, Janeza Trdine Rijeka, Croatia 2012; p. 9.

[8] Higuchi T, Lachman L. Inhibition of hydrolysis of esters in solution by formation of complexes III. Stabilization of tetracaine with caffeine. J Am Pharm Associ 1957; 46: 32-36.

[9] Andrews JL, Keefer RM. Molecular complexes in Organic Chemistry, London, Holden- Day, Inc 1964; p. 7.

[10] Vogel Al. A Textbook of Practical Organic Chemistry, $5^{\text {th }}$ ed. New York, John Wiley 1989.

[11] Weissberger A, Prokaher FS, Reddick JA, Toops EE. Techniques of Organic Chemistry, vol VII, Organic Solvents, New York, Interscience 1955.

[12] Baldevraj, Rajendran V, Palanichamy. Science and Technology of Ultrasonics, New Delhi, Narosa Publications 2004.

[13] Kannappan V, Jaya Santhi R. Ultrasonic investigation of induced dipole-induced dipole interactions in binary liquid mixtures at 298K. Ind J Pure Appl Phys 2006; 44: 815-19.

[14] Ishwara Bhat J, Manjunatha MN, Shree Varaprasad NS. Acoustic behaviour of citric acid in aqueous and partial aqueous media. Ind J Pure Appl Phys 2010; 48: 875-80.

[15] Ulagendran V, Kumar R, Jayakumar S, Kannappan V. Ultrasonic and spectroscopic investigations of charge- 
transfer complexes in ternary liquid mixtures. J Mol Liq 2009; 148: 67-72.

http://dx.doi.org/10.1016/j.molliq.2009.06.012

[16] Vibhu I, Singh AK, Gupta M, Shukla JP. Ultrasonic and IR investigation of $\mathrm{N}-\mathrm{H}$----- $\mathrm{N}$ complexes in ternary mixtures. J Mol Liq 2004; 115: 1-3.

http://dx.doi.org/10.1016/j.molliq.2003.08.024

[17] Awasthi A, Rastogi M, Gupta M, Shukla JP. Ultrasonic investigation of molecular association in ternary mixtures. $\mathrm{J}$ Mol Liq 1999; 80: 77-84.

http://dx.doi.org/10.1016/S0167-7322(99)00016-1

[18] Palaniappan L. Ultrasonic analysis in the ternary system of pyridine + benzene + 2-butanol. Physica 2008; B403: 388791.
[19] Kannapan AN, Kesavasamy R, Ponnuswamy V. Molecular interaction studies in the ternary liquid mixture of pyridine + benzene $+\mathrm{N}, \mathrm{N}$-dimethylformamide by ultrasonic velocity measurements. ARPN J Eng Appl Sci 2008; 3: 41-45.

[20] Mulliken RS, Pearson WB. Molecular Complexes - A lecture and Report volume, New York, Wiley Publishers 1969.

[21] Foster R. Organic Charge-transfer Complexes, London, Academic Press 1969; p. 387.

[22] Benesi HA, Hildebrand JH. A Spectrophotometric Investigation of the Interaction of lodine with Aromatic Hydrocarbons. J Am Chem Soc 1949; 71: 2703-2007. http://dx.doi.org/10.1021/ja01176a030

[23] Foster R, Ed. Molecular Complexes, London, Elek Science 1973; Vol. 1. 